\title{
Automated Decisions in Aware Cricket Ground: A Proposal
}

\author{
P. Ashok Kumar, Member, IACSIT
}

\begin{abstract}
The technology in the present world is an edge cutting in nature. The transformation of the technology is at various dimensions. Computing the data and the process of data and its interpretation is changing around the time in nature at its occupancy in the world. Computation of the data is transforming to a greater extent from the Grid to Cloud computing at high speed. The technology available today is not only a data processing one it has various dimensions and applications at which the performance of the these software's are achieving a higher accuracy and greater results. These technologies can also be implemented in sports which are a modern war fields. Games such as Football, Rugby, Hockey, and Cricket, Tennis and many other Team and single player games are being considered as prestige to their countries. The Judges or the umpires in those games have a great deal and value for their decision and sometimes their fault would turn the nature of the game. This research work is purely focused on the automated decision making in sports purely levering Ubiquitous Computing. Cricket is primarily a popular game in the eastern countries and now a more popular one after football is chosen for implementing this idea of automated decision making. The main objective of this paper is to achieve certain goals 1) Make a decision where a human error makes errors due to limitations. 2) Simulate the Match activity during and after the game in a 3D computerized Graphics system. 3) Make various types of game and performance analysis of a certain team or a player.
\end{abstract}

Index Terms-Decision making, Ubiquitous computing, Context Awareness, Sports, Cloud Computing.

\section{INTRODUCTION}

A person who follows sports like Cricket knows the value of the decision made by the umpire. At crucial situations in games a wrong decision makes the game an injustice to a team it has the same effect of winning and losing the game is spoiled, a big disappoint to the fans. Sports science makes the major parts of the game as activities such as practice during and after competition[1][4]. Computer-based and semi- automated observational system support coaches by combining quantitative statistics with qualitative video analysis [1]-[3]. A number of misjudged decisions by umpires due to incapability or simple carelessness led to totally spoiled matches. There are numerous examples which one can quote in this respect. "Test in England where an umpire gave three batsmen out on no-balls. TV replays showed that the bowlers had clearly violated the no-ball rule but no one called it [1], [2]. Past has many examples about these wrong decisions where the basic rules were also ruled out. In these decisions maximum

Manuscript received February 23, 2012; revised May 10, 2012.

P. Ashok Kumar is with Dept of MCA, SVIM, Tirupati, K. Ravali, Dept of IT, SVEC, Tirupati (e-mail: polisettyashok@gmail.com). part of the important decisions involve the decision to make weather a batsman was given out or not especially decisions such as LBW, RUNOUT, CAUGHT OUT, STUMPED. There are some decisions to be made at the bowler end also such as NOBALL, WIDE, BOUNCER. A series of cricket matches (VB Series 2005) played between West Indies, Australia and Pakistan teams, at the end Australia won but what happened? "Pakistanis claimed umpires favor vocal Aussies". Many other examples are there where game is affected badly due to wrong decisions of umpires and these decisions are not only taken once but repeated many times cautiously. There are some ODD decisions to be taken by the umpire in judging the BOUNDRIES and SIX and CATCHES take at boundary line. The fielding rules are also to be watched out by the umpire.

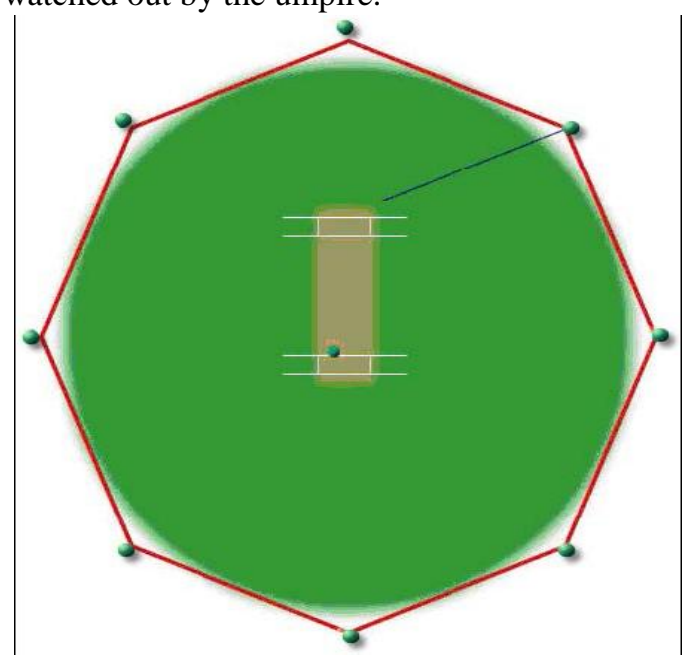

Fig.1. Placement of Access Points

There exist a number of problems which cause dissatisfaction of the players and game lovers when a decision is of great importance and a misjudgment done by an umpire crumbles the whole situation. The Automated ball tracking system proposed in this paper helps solve a number of key issues in judgment and decision making during the game using ubiquitous computing[1]-[3]. Problems addressed in this paper are:

1) No Ball Decision

2) Stumping Decision

3) Leg Before Wicket Decision

4) Wide

5) Position of Fielders in Restricted Overs

6) Runout

7) Boundry Line Decision

8) Critical Catches

A number of other useful information can also be extracted from the data collected by the system.

- Bowling Speed 
- Fielding Analysis

- Striking Analysis

- Strike Power etc.

The structure of our paper is organized as follows:

Section 2 reviews the related literature. Section 3 presents our proposed system. In Section 4 our proposed system is compared with the existing system and Section 5 concludes the paper and section 6 describes the Future work \& Conclusion.

\section{RELATED WORK}

A very little work is done in this area, thus new tools and systems are required for supporting all kinds of human activities. The systems must have the goals of accuracy, robustness and Security. A brief detail of the existing work is described below:

M. Beetz et al. [1], [5]-[7] have developed the Football Interaction and Process Model FIPM and a software system that can acquire, interpret, and analyze this model. The system can acquire models of player skills, infer actionselection criteria, and determine player and team strengths and weaknesses. This system extracts the events of the game based only on the position of players and the ball. It has been implemented using the microwave sensors mechanism for automatic analysis of the football during the game. The FIPM game analysis system assumes a real-time positioning system that can continually track the positions of the feet of all players and the ball with an accuracy of a few centimeters and provides position data for the ball that enables the game analysis system to accurately detect ball contacts of the players.

Jonathan Hey and Scott Carter[1]-[9] have designed and implemented an enhanced table tennis practical table called Memory Tennis which projects a lasting image of the last place the ball struck on the vertical practice wall. Using this, the players can determine their accuracy immediately and actively compensate for poor shots. Additionally, players can get statistics on past performance. The system can project targets for players to hit and act as coaching program as player's performance improves.

A. G. Foina et [1]-[10] al have designed a tool to help sports coaches to analyze their players using an RFID technology connected to 3-layer software. The system has two tracking modes, one for 2D player location in the field and a 3D mode to capture player's movement in small area sports. It has two report modes also, one for real time report displaying each player's actual location in the field and the other to present reports with statistics of the player. Zoe Drey and Charles Consel [1]-[8] have presented a visual programming language called Pantagruel which is end-user oriented. Their approach is open ended in that Pantagruel integrates a language to describe a ubiquitous computing environment. To facilitate the programming of the orchestration logic, they have developed a visual tool that uses a sensor-controller-actuator paradigm. An Orchestration logic collects context data from sensors, combines them with a controller, and reacts by triggering actuators. They have assessed the usability of this paradigm with a successful user study conducted with 18 nonprogrammer participants. Furthermore, their visual programming environment offers the developer an interface that is customized with respect to the environment description. Ed H. Chi et al have propelled ubiquitous computing into the extremely hostile environment of the sparring ring of a martial art competition. A novel wearable computing system called True Score TM Sensor Hogu for supporting an extreme human activity namely Taekwondo competition sparring. It uses piezoelectric sensors to sense the amount of force that has been delivered to a competitor's body protector and wirelessly transmits this signal to a computer that scores and displays the point. Their objective is to support the judges in scoring the sparring matches accurately while preserving the goals of merging and blending into the background of the activity.

Ramy Asselin [1]-[13] et al have presented the design and evaluation of their proposed system called Personal Wellness Coach System (PWC). It supports a range of activities including health data collection, interpretation, and feedback and self-monitoring. They have implemented a wearable computing platform which consists of an array of sensors and application software to motivate users to reach fitness goals and prevent harm in a real time environment.

\section{PROPOSED SYSTEM}

This paper focuses on finding the location of the ball during the game at any given instance and thus making the desired calculations whenever needed for a certain analysis. The System makes decision making it a very easy and painless task. It also makes it possible to animate the whole game at any time in a 3D Computer animation. It is a high precision system and a bulk data producer, it can be used to make a vast variety of decisions based on this information [1]-[4].

\section{A. Working of Proposed System}

Sensors are placed at various hotspots in the playground, cricket ball, and in the shoes of the players. With the help of these sensors information about all aspects of the game is collected and sent to a central computer for further processing. The Computer collects the data, formats it for storage and stores it in a ways that it can be used for any purpose in the future. Data received from various sensors placed in the moving objects is processed and we get the distance of object from certain predefined point. Using the same mechanism we address the problems in the order as mentioned above.

\section{1) No Ball Decision}

We only address the issue of over stepping the popping crease by the bowler. To detect whether a specific delivery is valid or is a no ball. We use a triangular placement of sensors as shown in Fig. 2.

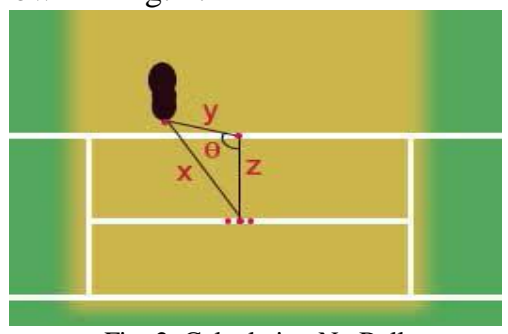

Fig. 2. Calculating No Ball 
We achieve a triangular assembly of sensors by mounting two sensors statically in the crease and the third one in the shoe of the bowler. Sensors in all the 3 positions talk to the central computer and communicate their $(x, y)$ coordinates. Once we have the coordinates of the sensors we can calculate the length of the sides of the triangle using distance formula as given in equation (1)

$$
d=\sqrt{\left(x_{2}-x_{1}\right)^{2}-\left(y_{2}-y_{1}\right)^{2}}
$$

The distance formula provides the lengths of all the three sides of the triangle and we can calculate the angles involved in the creation of the triangle using the formula given in the equation (2).

$$
x^{2}=y^{2}+z^{2}+2 y z \cos \theta
$$

Here a check is made before calculating the angle, as we know that the length of the side which is labeled $\mathrm{z}$, now if the side $\mathrm{x}$ gets smaller in length then the side $\mathrm{z}$ it give an automatic judgment of not being a no ball. If the angle $\theta$ is greater than $90^{\circ}$ then it is assumed that the bowler has over stepped the popping crease line, indicating a no ball. Once a no ball is detected the system alarm for no ball and communicates it to the umpire as well as to the scoring system [1]-[5].

\section{2) Stumping Decision}

The stumping is a type of Dismissal of a batsman in the game of cricket. A batsman is dismissed even for a No-Ball also. This issue can also be addressed similarly by the above issue No-ball [1]. Generally stumping is done at the strikers end by the wicketkeeper, whenever the batsman misses the ball when he moves forward to strike the ball. Generally these type of decisions are little critical to the Leg umpire when they meet at the time-line and the $3^{\text {rd }}$-Umpire might be helpful but at close calls it is a benefit of doubt which make situation a false on to either of the sides. This can be avoided by keeping sensors in the batsman shoe i.e. when the batsman moves forward to play the shot and if he misses or when he attempts to hit the ball and misses it, if the wicketkeeper is close to the wickets he can collect the ball and attempt to stump the batsman at that time the batsman is considered out if the keeper hits the ball with the ball in his gloves and if the batsman is outside the crease. This makes us that the decision can be made false if any of the condition is failed. Our system can suggest the umpire and make decision that the batsman is out or not in the following manner.

Whenever the keeper collects the ball and hits the stumps with the ball in the hand ours system would check the sensor of the ball and the wicket are met such that it confirms that the ball had connected to the wicket and at the same time our system also checks the distance between the batsman foot and the wickets similar to the bowlers foot in the No-ball with the above Equations-1\&2. Here a check is made before calculating the angle, as we know that the length of the side which is labeled $\mathrm{z}$, now if the side $\mathrm{x}$ gets smaller in length then the side $\mathrm{z}$ it give an automatic judgment of not being out. If the angle $\theta$ is greater than $90^{\circ}$ then it is assumed that the batsman has been out of the crease line, indicating stumped. Once this is detected the system alarm for stumped and communicates it to the umpire as well as to the scoring system[1]-[6].
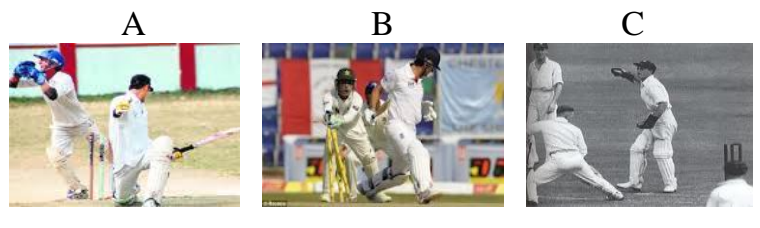

Fig. 3. Images of Stumping in cricket at various stages

In the above 3 images $\mathrm{A}, \mathrm{B}, \mathrm{C}$ they represent the stumping of a batsman at times in the game these are a little critical thing to be pointed by the Umpire in the ground due to various reasons. Our system provides accurate decision at any point of time. A Scenario is described below here the decision made even by the $3^{\text {rd }}$-umpire may also be wrong.

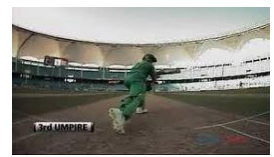

Fig. 4. Mid-Stump Camera View Image

In the above image the bails were in the sky but the batsman foot was not been identified in or out /on the crease line. By our Equations we can provide a right decision.

\section{3) Position of Fielders in Restricted Overs}

We know that sensors have already been mounted in the shoes of all the players and they are in constant contact with the central computer, so it is quite easy to calculate the number of fielders in any location of the cricket ground. The below image is a predicated output of various fielders in the ground.

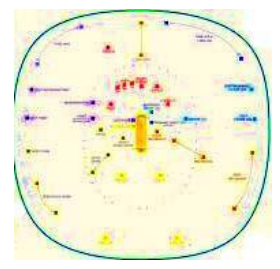

Fig. 5. Position of Fielders in the ground

In the above image the fielders are shown with various colors such as Red, Yellow, Blue, and Violet. These are the various positions of the fielders and at different situations of the game. The points resemble the fielder's positions and there positional movements are can be observed with the lines. This can be used as Fielding analysis in the ground at various intervals of time.

\section{4) Wide}

Generally a Wide is a mistake done by the bowler in the game of cricket like a No-ball. A Delivery is called wide by the umpire when the ball travels away from the batsman crossing the amount of width from the stumps. Generally this decision is looks to be simple but at times it is also crucial. Our proposed system calculates the delivery is wide or not. When the ball is delivered and if the batsman does not have any contact with the ball and if the ball passes 
away from the specified distance then we can decide that the ball is a wide. To calculate the ball is a wide or not we require few parameters in making the decision.

1) To which handed batsman is the ball being delivered?

2) What is the position of the ball from the Mid-stump?

3) What is the distance between its position and Midstump?

From all these parameters we can make a decision and decide whether the ball is a wide or not. In cricket there are two types of batsman Left handed and right handed. If the ball is bowled to the leg side of any of these batsmen then we can declare it as a wide. When the first parameter is identified then we move to the next parameter, if the ball position is towards the leg side in either of the batsman then without checking the third parameter we can declare it as a wide. If the ball is not bowled to the led side and still if the ball is moving away from the stumps i.e. after identifying the first two parameters we calculate the distance from these two points using the Eq (1) and Eq (2) and then if the distance is greater or equal to the allowed width then we can declare it as a wide. A wide is not only the distance away from the stumps it can also be the height above the stumps which sometimes labeled as a No-ball or wide. This is a choice to be made by the umpire during the match. In this way our proposed system makes decision either the ball is a wide or not.

\section{5) Leg Before Wicket Decision (LBW)}

\section{a) Trajectory Calculation:}

The Trajectory calculation for Leg-Before Wicket till today has been achieved through the use of Hawk eye camera and other similar image processing and computer vision technique using cameras mounted for capturing the match events. We are deploying different mechanism for calculation of the ball trajectory. We have a special type of sensor which responds to the nearby microwave radar, as the moving ball communicates its position to the receptor once every 100th of a second. So we have quite enough amounts of data about the movement of the ball, which can be used to calculate the parabolic curve of the ball traveling across the wicket.

Trajectory calculation can be precisely done on the basis of points obtained from the previous motion of the ball in the prescribed plane.
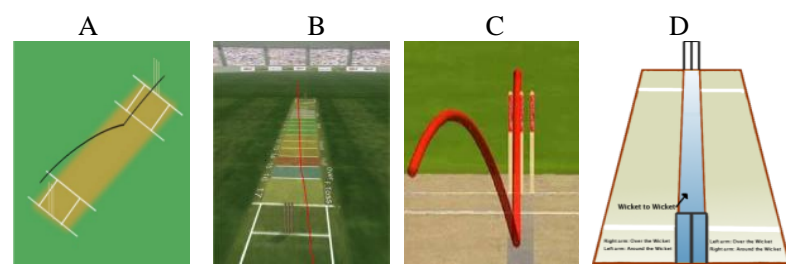

Fig. 6. Hawk-Eye Trajectory Images

In the above images A- is the image obtained from our trajectory calculation of the ball. B-image is also represents the same but it also specifies the area in which the ball had been placed and its movement towards the batsman end. Cimage is the complete trajectory calculation with the height the ball had bounced or the height the ball had been from the ground when it reached the batsman. D-is the image which represents the path between the wickets generally called as wicket to wicket.

If the ball pitches in that path and if the ball hits the batsman pad and then the umpire can calculate the impact of the ball and can decide the batsman is out or not. It cannot be assumed to be accurate at all the times.

\section{b) Decision using the sensors:}

The trajectory is an excellent method for making decisions but there are at situations where the trajectory may be mis-interpreted and a wrong decision can be implied to the batsman because the batsman is not out if the ball pitches in the line and hits the pads. There are some other factors to be considered such as
1) Where did the ball hit the Batsman?
2) What is the height of the ball?
3) What is the trajectory of the ball?
4) What is the position of the batsman?

These are some important factors to be considered by us when we make the decision. The below image shows the various ways the ball had hit the batsman pads travelled in or out the path i.e. Wicket to wickets path. In the trajectories of $\mathrm{A}, \mathrm{B}, \mathrm{C}, \mathrm{D}, \mathrm{E}$. which are travelling to the wickets. According to Ball Trajectory all of these actions resemble us that the batsman was out. Only the Balls B, C, $\mathrm{D}$ are considered as LBW as they tend to hit the wicket if the batsman misses the ball.

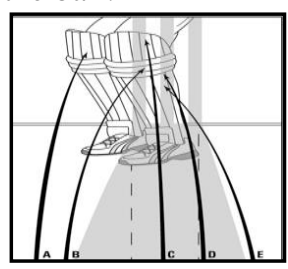

Fig. 7. Various trajectories of the ball resembling LBW

Accurate Decision can be made by us by using the sensors available in the ball, wicket, batsman shoes, pads and bat. Whenever the ball hits the batsman pad our proposed system observes various factors before making a decision such as:

1) When the ball hits the batsman pads first our system identifies weather the ball had hit the batsman pad directly?

2) Did the delivery is a correct one?

3) Did the ball travelled in the path before it hit the batsman pads?

4) What is the deflection of the ball from the Midstump?

5) What is the Height of the ball?

6) What is the position of the batsman foot when the ball had hit the batsman pads?

These questions are answered by our system by which our decision is made easier and accurate. Our system answers the first factor by detecting weather the ball had any contact with the bat. It is processed with the sensor available in the bat and ball, if they are connected there is a 
signal informing us that the ball had connected with the bat it is nothing but the deflection obtained from the bat sensor. The $2^{\text {nd }}$ factor is answered by our No-ball decision system which communicates to us if it is a No-ball. The $3^{\text {rd }}$ and $4^{\text {th }}$ factor are calculated by the system with the Distance between the 2 points i.e. using Equation 1 and 2. Let the Sensor in the wicket is recorded as $\mathrm{X}$ and the place where the ball had landed in the pitch is $\mathrm{Y}$ now we calculate the distance and deflection between these two points and another point $\mathrm{Z}$ is considered where the ball had hit the batsman pad using the ball sensor now again using the Equations-1 \&2 we calculate the distance and Deflection of the ball with respect to the mid -stump.

The value $\theta$ will provide us that the ball is deflecting either right or to left at first position $Y$ to $X$. Again the $\theta$ value is calculated for the points between $\mathrm{X}$ and $\mathrm{Z}$ this deflection provides us that weather the ball is moving away from the stumps or into the stumps and we can also calculate that the ball will hit the stumps or not. The $5^{\text {th }}$ factor is also addressed by the system by considering the sensor of the stumps and the impact of the ball that had hit the stumps. Again by using the Equation-1 we can get the Height of the ball and if the height is less than the height if the stumps we can assume that the batsman might be out by considering the various factors. The last factor can be addressed by our system by pointing the batsman position when the ball had hit his pad and also the position of the foot where the ball had hit the pad. We can also calculate the distance between the Foot and the stumps to identify that the batsman was in the line or crossed the line.

By considering the above 6 factors we can make a decision in 3 steps:

1) If the system output is 1 i.e. ball had directly hit the batsman pad and the ball is not a No-ball.

2) Here again if we get the output from the system as 1 i.e. the ball had been in the path and the deflection is into the stumps and the height of the ball when it hit the batsman pad is below the height of the stumps.

3) If the output is again 1 from the system i.e. the batsman position is in the path and near to the stumps.

If the output from the 3 stages in ONE then our system declares that the batsman is out. Both the systems provide accurate decisions.

\section{6) Runout}

The most common way where any one of the batsman at the ends can get out. A run out is accepted even for a NoBall. The run out decision generally in the game of cricket is made by the umpires in the field and the third umpire also. At some stages in the game the run outs are made to be crucial decisions for the umpires to make a decision. For an effective decision to be made by an umpire he requests the $3^{\text {rd }}$ umpire and he gives the decision by using the cameras available round the ground and in the ground. This is also not an easy task to be considered by the $3^{\text {rd }}$ umpire as the technology may not be helpful in making clear decisions. These issues can also be answered by our system in affective manner. The input required to make the decision are:

1) Our system has to record the Batsman sensors available in their shoe and bat.

2) If any substitute is provided for the batsman his sensors are to be recorded along with the other people.

With these inputs loaded into the system and other inputs such as sensors available in the ball, wickets, bowlers and other player's sensors are used in making the decisions. To differ stumping from run out 3 sensors are placed along the pitch where the batsman has to run. 2 sensors are placed away from 5 yards to the stumps at both the ends. Another sensor is placed in the halfway of the pitch to record that the batsman had attempted to run. The system makes decisions in the following steps:

1) When the ball hits the stumps by any player in the ground our system detect from where the ball is thrown.

2) If the ball is thrown by any player other than bowler and if the batsman bat or leg is not grounded in the crease at any end the batsman running at that end is considered as run out.

3) If the bowler makes the attempt it has a certain criteria to make the decision.

In the above described 3 steps our proposed system makes decisions. The $1^{\text {st }}$ is used by the system to record which fielder had thrown the ball to the keeper /bowler. The $2^{\text {nd }}$ step is that when the ball connects to the stumps if the batsman leg or bat must be in the crease. This is calculated using the mid-stump sensor and batsman leg/bat sensor. When the bowler/keeper collects the ball from fielder and hits the ball to the stumps at any end, the sensor of the ball and the stump will get connected that is considered as the input to make decision. At that time in that end the batsman sensors of leg $\&$ bat both distances are calculated using the Equations-1\&2 if both the distances are greater or equal to the distance of the crease the batsman running to that end is declared as Run out. If the ball doesn't connect to the stumps even if the batsman is out of the crease he is considered as not out. The distance of the sensor is calculated when they are grounded only. The $3^{\text {rd }}$ step is a special case i.e. if the bowler attempts to out the batsman at non-striker end without delivery or on a straight drive occasion. In these 2 situations there are chances where the non-striker is out. In the first situation i.e. when the bowler attempts to deliver the ball and if he attempts to out the batsman the ball has to be touched to the ground and then he has to hit the stumps. Here we would weather the ball had touched the ground by using the sensor available in the ball. If the batsman is away from the crease he is out. If the batsman drives the ball and if the bowler collects the ball and hit the stumps and if the non-striker is away from the crease he is declared as out. Here our proposed system would check whether the ball had any contact with the bowler and hit the stumps. It can be identified by the system using the sensor in the ball and the deflections of the ball. The Proposed system declares a batsman as run out if the batsman bat and leg sensors distance is greater or equal to 
the distance of crease to stumps. If any of it is shorter he is considered not out only when the batsman leg / bat sensor are recorded at the 3 sensors this is considered when the batsman misses his bat while running.

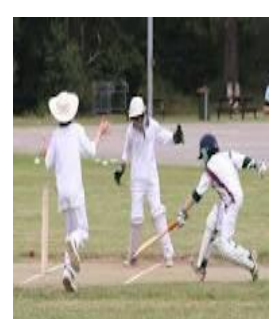

A

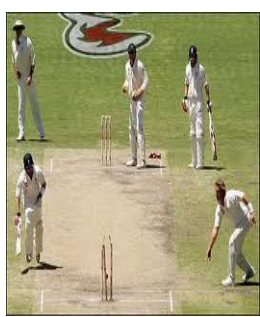

B

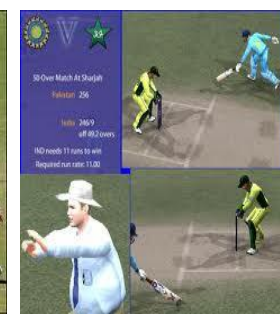

$\mathrm{C}$
Fig. 8. Run Outs At Various Situation In Game

In the above 3 images $\mathrm{A}, \mathrm{B}, \mathrm{C}$ we can identify the 3 cases where the batsman were out. In A the batsman is made run out by the fielder by directly hitting the stumps before the batsman makes it ground. In B when the batsman attempts the shot and then the non- striker attempts to run where the bowler collects the ball quickly and throws the ball to stumps and it hits the stumps and he is declared as Run Out. In the image $C$ the batsman is attempting to ground the bat in the crease before the wicket keeper collects the ball and connects it to stumps and we can see the umpire declaring it as not out. The image is taken form then famous computer Game CRICKET-2007 developed by EASPORTS. In this manner our proposed system also makes decisions at an accurate phase.

\section{7) Boundary Line Decision}

The boundary line decision also a crucial decision for the umpire to be taken at times in a match. Generally in the game of cricket the boundary line is the outer line of the cricket ground generally placed far away with a distance around 90meters from stumps. So the decision whether the ball had gone to the boundary or not is an issue to be discussed in some scenarios of the game. When the batsman strikes the ball and if the ball had touched the boundary line or if the fielder who is fielding in the ground makes contact with the ball and the boundary line it is to be considered as a boundary. At times the chase might end with a close call where the fielder might have contact with the ball as well as the boundary line. The third umpire might not be able to make a clear decision. If we place sensors around the boundary line our proposed system can provide us the accurate decision. The issue can be answered by our system in the following manner:

1) If the ball gets contact with the boundary line it is declared as a boundary. This is known to our system with the sensors available in the ball and round the ground if the ball contact with the boundary line, our system immediately passes the message to us.

2) If the fielder had made contact with the boundary line then our system passes the message to us and if the fielder dose have contact with the ball during that point of time our system passes us that the ball, fielder are in contact with each other and the fielder is in contact with the boundary line hence they are connected so our system passes message to us. The decision is made in 2 phases during the initial phase we identify weather the fielder or the ball had any contact with the boundary line using the sensors available in the ball and the fielder. If nothing is in contact it is not a boundary. If there is a contact then in the next phase our system identifies if the contact is made by the ball or by the fielder. If it is the ball our system declares it as a boundary. If it is the fielder our system checks the position of the ball either it is in contact with the fielder or not if yes it can be assumed as a boundary. If not it is passes a message as not a boundary. This decision is particularly a critical one during the day and night matches or at times during the game.

\section{8) Critical Catches}

Catches are an important segment in the games like cricket. A famous quotation is also made by legends of cricket as "Catches Win Matches". That is the important of a catch which can change the course of the match. At times the decision becomes critical for the field umpires and also to the third umpire. The catches that are taken near to the ground called as Dive catches which are critical to judge. Our system solves this problem in a much simpler manner. Whenever the umpires request the system our system checks weather the ball had made contact with the ground or not if not, the decision is out else not out. Our proposed system will make the decision by using the sensor available in the ball if the ball had made contact with the ground our system will identify it easily. In this manner our proposed system will help the Umpires in the game to make accurate decisions.

\section{B. Proposed System Architecture}

Our system is organized as a four -tier layered architecture according to their functionality, which is given under.

1) Data Generation/Data Gathering Layer:

The Sensor/ hardware (camera's etc) will sense/ capture the event and generate the data.

\section{2) Data Communication Layer:}

The generated data is collected from sensors and other different Hardware (i.e. camera's etc) through the network of an inter-connected access points and cables and is transferred to the Main Software processing Unit.

\section{3) Data Processing Layer:}

The main software will process the collected data and transfers the results.

\section{4) Data Presentation/Data Reporting Layer:}

Processed Data will be used/ presented for further actions according to user request like:

- $\quad$ For umpires in making a decision.

- For coaches to analyze the game and future planning. 
- $\quad$ For viewers in making analysis \& statistics.

The stored data can be used for future analysis by the players and team management to improve their game, and overcome their weaknesses. It also finds out the weakness/strengths of other players[1]-[8].

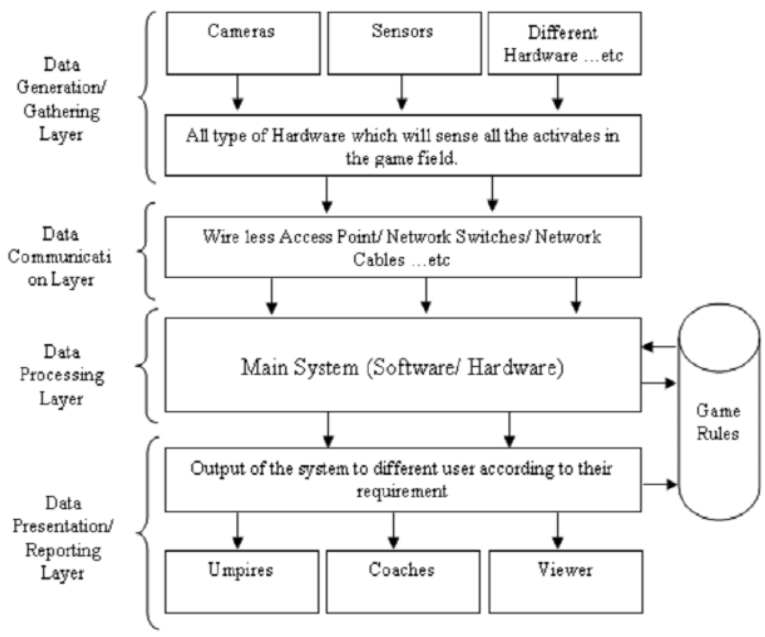

Fig. 9. Proposed System Architecture

\section{ADVANCEMENT IN THE PROPOSED SYSTEM}

The proposed system had 4- layers as shown in above figure. The proposed system can be advanced by implementing the cloud computing technology in Data Communication Layer by which the Ubiquitous Computing is shifted to Cloud computing. The cloud computing is an emerging technology in the Information technology. The cloud computing enables to make the system as Software as a Service (SASS) and the game rules can be provided to multiple group end users such as Umpires, Coaches, and viewers[1]-[14]. The proposed system is modified a little with the cloud computing and the modified proposed system is shown below. The Data Communication Layer is replaced with the Cloud image i.e. Cloud Computing which acts as a medium for Data Communication. This proposed system had more advantages than the other system. By introducing the Cloud the same system can be used for multiple purposes.

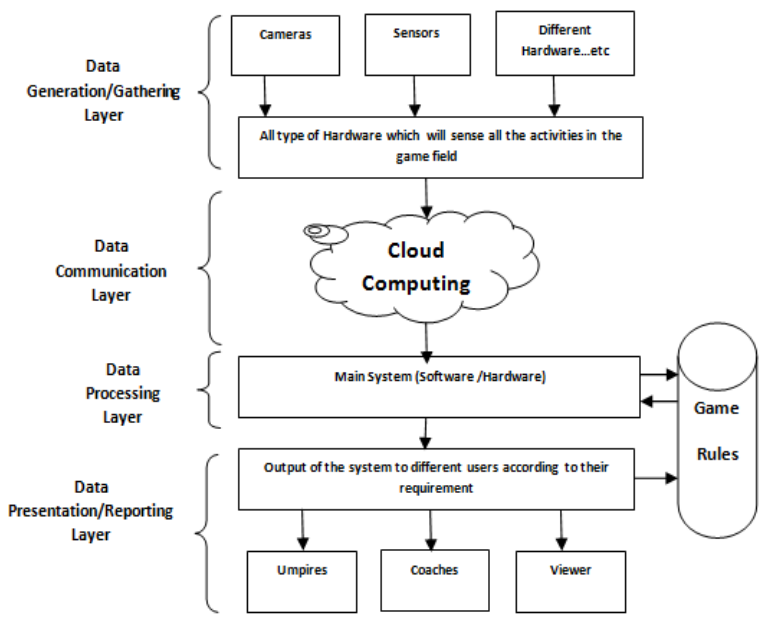

Fig. 10. Proposed System Architecture With Cloud Computing

\section{COMPARISON WITH EXISTING SYSTEMS}

Currently for decision taking according to the cricket rules, umpire's decision is final but however if there is any doubt, the ground umpire can take help from the third umpire who uses recorded videos for giving his final decisions. The Decision review System (DRS) is a new feature introduce in international cricket is advancement but it is a controversial thing and limited to a less number of decisions which may also be a loss to the spirit of the game many software's are available which can calculate the trajectory of the ball. Hardware like speed calculation cameras are available which can calculate the speed of the ball. Factors like wind speed, wind directions, humidity in air, pitch condition, ball's conditions can determine the direction of the ball. Some software can also help in determining statistical analysis and scoring the game.

All the available techniques for checking different activities of cricket work independently. Comparatively, our proposed system works in a combined fashion assisting in correct decision making.

It considers all the important activities of the game which if not considered during decision making may spoil the whole game.

\section{CONCLUSION \& FUTURE WORK}

The proposed system has provided an advantage in making accurate decisions at critical times of the game. It lifts the Spirit of the game may more new tools with the advancement in the technology can produce more tools by which the future challenges such as tracking the ball in real-time, sensing the no ball etc Accuracy and in -time detection of problems of the outdoor games can be addressed which is also a big challenge for the researchers.

Many areas in the outdoor games require the support of various system which are proposed above, these systems automates many areas in the game. These systems are more helpful for the Umpires, coaches, and viewers of the game. A Fuzzy database can also be used by us in future for making decision in which the Artificial Intelligence can be used for all the sort of decisions where the system takes about the decisions in the game which replaces the human umpire and makes decisions much more accurately. By using the fuzzy logic many critical decisions are made simpler and the system performance is at its best in making decisions. We hope the future decisions are shaped the machines which use the Artificial intelligence, Neural Networks and Cloud computing etc. emerging technologies and enhance better decision making in all sort of games and in many more areas.

\section{ACKNOWLEDGMENT}

We wish to acknowledge the anonymous reviewers for their valuable comments.

\section{REFERENCES}

[1] W. Z. Khan, Md. Y Aalsalem, and Q.Ain Arshad: "The Aware Cricket Ground," IJCSI Issues, vol. 8, issue 4, No 2, July 2011

[2] M. Weiser: "The Computer for the 21st Century," Scientific American, vol. 265, no.9, pp. 66-75, 1991. 
[3] A. Hohmann, M. Lames, and M. Letzelter, Training swissens chaft The Science of Coaching, in German, 2001.

[4] M. Lames and G. Hansen, "Designing observational systems to support top -level teams in game sports," International Journal of Performance Analysis, vol. 1, pp.85-91, 2001.

[5] M. Beetz, F. Fischer, S. Flossmann, B. Kirchlechner, A. Unseld, and C. Holzer, "Watching football with the eyes of experts: Integrated intelligent systems for the automatic analysis of (simulated) football games," in 5th Annual Conference dvs -Section Computer Science in Sport, 2004.

[6] M. Beetz, S. Flossmann, and T. Stammeier, "Motion and episode models for (simulated) football games acquisition, representation, and use," in 3rd International Joint Conference on Autonomous Agents \& Multi Agent Systems (AAMAS), 2004.

[7] M. Beetz, B. Kirchlechner, and M. Lames, "Computerized real-time analysis of football games" Pervasive Computing," IEEE, vol. 4, issue 3,.pp. 33 -39 July-Sept 2005.

[8] Z. Drey and C. Consel, "A Visual, Open -Ended Approach to Prototyping Ubiquitous Computing Applications," IEEE, pp. 817-819, 2010.

[9] J. Hey and S. Carter, "Pervasive computing in sports Training," IEEE CS and IEEE ComSoc, 2005.

[10] A. G. Foina and R. M. Badia, A. EI-Deeb, F. Javier, RamirezFernandez "Player Tracker - A Tool to Analyze Sport Players using RFID," IEEE, pp. 772-775, 2010.

[11] Z. Drey and C. Consel, "A Visual, Open-Ended Approach to Prototyping Ubiquitous Computing Applications," IEEE, 2010, pp.817-819.

[12] Ed. H. Chi, J. Song, and G. Corbin, "Killer App-of Wearable Computing: Wireless Force Sensing Body Protectors for Martial Arts," ACM, vol. 6, issue 2, 2004

[13] R. Asselin, G. Ortiz, J. Pui, A. Smailagic, and C. Kissling, "Implementation and Evaluation of the Personal Wellness Coach", in
Proceedings of the 25th IEEE International Conference on distributed Computing Systems Workshops (ICDCSW'05),2005.

[14] The NIST Definition of Cloud Computing (Draft), National Institute of Science and Technology. Retrieved 24 July 2011.

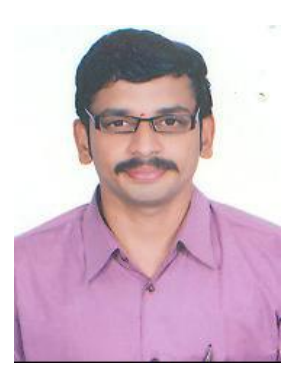

Ashok Kumar Polisetty received his MCA in 2008 from Osmania University, India. $\mathrm{He}$ is currently pursuing his P.hD from Dravidian University in the area Cloud Computing. $\mathrm{He}$ worked as Assistant Professor in Dept of MCA, in Laqshya College of Computer Studies, Khammam. He is currently working as Assistant Professor in Sree Vidyanikethan Institute of Management, Tirupati. His current areas of research include Cloud Computing, Data Mining, Image Processing, Decision Making and Information Security. He is reviewer for IAENG, IJCTA ,JCER, IJITCS Journals. He is a member in IAENG, IACSIT.

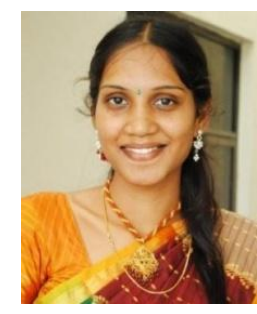

Ravali Kota received her B.Tech Degree in Information Technology in 2009 from JNTU, Hyderabad, Andhra Pradesh, India. She worked as Assistant Professor in Laqshya Institute of Technology \& Science, Khammam from 20092010. She is currently working as Assistant Professor in Sree Vidyanikethan Engineering College Autonomous, Tirupati. Her current research interests are Data Mining, Green IT, and Cloud Computing. She is a member in IAENG. 